\title{
ALGORITMOS GENÉTICOS E VARIANTES NA SOLUÇÃO DE PROBLEMAS DE CONFIGURAÇÃO DE REDES DE DISTRIBUIÇÃO
}

\author{
E. P. Bento* \\ N. Kagan* \\ *ENERQ - Centro de Estudos em Regulação e Qualidade de Energia - Escola Politécnica - USP \\ Av. Prof. Luciano Gualberto, trav. 3, n. 158, CEP 05508-900 - São Paulo - SP
}

\section{RESUMO}

Este artigo apresenta uma metodologia para resolução de problemas de configuração de redes de distribuição, com aplicação à minimização de perdas elétricas. O método utiliza um Algoritmo Genético (AG) básico e algumas de suas variantes para seus operadores genéticos: Seleção, Cruzamento e Mutação. O emprego do AG possibilita a análise de redes reais, sem necessidade de simplificações ou aproximações, o que permite a obtenção de soluções otimizadas em tempos de execução compatíveis para aplicações em atividades de planejamento da expansão e da operação. As variantes do AG permitem melhorar a robustez dos resultados e reduzir os tempos de execução, compatíveis com a aplicação em redes de distribuição. A metodologia proposta é aplicada, inicialmente, a uma rede de distribuição hipotética, onde é conhecida a configuração de perdas mínimas. Diversas alternativas de parametrizações do AG são efetuadas, o que permite a comparação do desempenho de cada alternativa. As melhores alternativas são aplicadas na resolução de uma rede real, mostrando o grande potencial do modelo.

PALAVRAS-CHAVE: Minimização de perdas elétricas em redes de distribuição, Otimização, Algoritmos Genéticos.

\section{ABSTRACT}

This paper presents a methodology to evaluate the optimal

\footnotetext{
Artigo submetido em 28/02/2005

1a. Revisão em 07/08/2006

2a. Revisão em 14/06/2008

Aceito sob recomendação do Editor Associado

Prof. Ivan Nunes da Silva
}

configuration of radial electric power distribution systems. Network loss minimization is set as the main objective to demonstrate the potential in solving real problems. The proposed framework is based on genetic algorithms (GA), which makes possible the analysis of real sized networks. This allows for attaining optimized solutions in an affordable computation time, especially for expansion and operation planning applications. The method requires neither simplifications nor approximations to the original problem formulation, thus improving the quality of the obtained results. Basic (or canonical) GA and some variants are presented, highlighting main functional characteristics of each alternative and respective parameterization. The proposed methodology is first applied to a hypothetical network, in which the minimum losses configuration is known in advance. Several simulations make possible comparing the performance of each GA parameter. Finally, the best parameter settings are applied to a real sized network, illustrating the promising potential of this methodology.

KEYWORDS: Losses minimization, Distribution networks, Optimization, Genetic algorithms.

\section{INTRODUÇÃO}

Propõe-se neste trabalho uma metodologia para a determinação da configuração ótima de redes de distribuição objetivando a minimização das perdas ativas, utilizando Algoritmos Genéticos (AGs). Este problema foi tratado por diversos autores. (Nara et alii, 1992) e (Miranda et alii, 1994) foram os precursores na aplicação de AGs para a resolução deste tipo de problema. (Kagan, 1999) aprofunda a utilização 
da técnica para aplicação em redes reais, utilizando conjuntos fuzzy para o tratamento de incertezas e aspectos subjetivos da tomada de decisão. Outro trabalho que determina a configuração de mínimas perdas, com demandas variáveis, é apresentado por (Cebrian, J. et alii, 2003), com critérios para variação das taxas de recombinação e mutação do AG. Trabalhos mais recentes apresentam pequenas variações, principalmente com alguns detalhes de variações no GA básica e na codificação do problema (Enacheanu et alii, 2008).

Neste trabalho, o principal objetivo é a análise de diversas variantes do algoritmo genético, como diferentes métodos de seleção (proporcional ou "roleta", torneio e ranking, com opção elitista) e cruzamento (um ponto, dois pontos, uniforme e uniforme com controle estatístico) e controle dinâmico sobre a probabilidade de mutação, aplicado aos indivíduos da população.

Os desempenhos das diversas variantes analisadas do AG são comparados com o AG básico mostrando como estas variantes permitem maior eficiência do processo, alcançando-se soluções otimizadas e em menor número de gerações, em função do problema a ser tratado e da codificação utilizada para o algoritmo genético. A utilização de codificações melhores, isto é, mais adaptadas ao problema, tende a levar os AGs a obterem soluções melhores em menor tempo de processamento.

Para a realização destas comparações, em termos de tempos de processamento e soluções encontradas, uma codificação eficiente é utilizada, baseada em blocos de carga (Kagan, N., 1999), que permite a avaliação de somente soluções radiais e conexas, diminuindo o espaço de soluções possíveis a serem pesquisadas e, conseqüentemente, aumentando a eficiência do algoritmo.

\section{OTIMIZAÇÃO DE SISTEMAS}

Problemas de otimização são freqüentemente encontrados em diferentes campos técnicos, como Engenharia, Economia, ou mesmo no nosso dia-a-dia. Freqüentemente, os problemas almejam objetivos contraditórios, relacionando custos, prazos, lucros, qualidade, eficiência e outras variáveis.

A formulação de um problema de otimização qualquer envolve a composição de uma função-objetivo, que relacione as diferentes variáveis consideradas, bem como as restrições impostas a cada uma. Otimizar um determinado problema consiste em identificar a solução, ou os respectivos valores para as variáveis consideradas, que maximize ou minimize o valor da função-objetivo, conforme a natureza do problema, de forma que nenhuma outra solução atribua um valor maior ou menor à função, respectivamente, respeitadas as restrições do problema.
Formalmente, as diferentes técnicas de maximização de uma função objetivo $f: M \subseteq \Re^{n} \rightarrow \Re$ buscam identificar uma solução, em particular $\vec{x}^{*} \in M$, tal que $\forall \vec{x} \in M: f(\vec{x}) \leq$ $f\left(\vec{x}^{*}\right)=f^{*}$, onde $f^{*}$ é um máximo global de $f$, obtida a partir da modelagem de um problema real, de complexidade e dimensão variáveis. Os problemas de minimização podem ser considerados problemas de maximização, onde $\operatorname{Max}\{-f(\vec{x})\}=\operatorname{Min}\{f(\vec{x})\}$.

Com freqüência, os problemas reais apresentam máximos locais, que introduzem dificuldades adicionais aos diferentes algoritmos de otimização existentes. Máximos locais são pontos no espaço de soluções $f^{\prime}=f\left(x^{\prime}\right)$ com valor significativamente maior que os demais em sua vizinhança, mas ainda inferior ao máximo global da função objetivo. Nestes casos, $\exists \varepsilon>0, \forall \vec{x} \in M:\left|\vec{x}-\vec{x}^{\prime}\right|<\varepsilon \Rightarrow f(\vec{x}) \leq f^{\prime}<f^{*}$.

Problemas de otimização com restrições consideram limites para as variáveis, definidos por um número $n_{\text {restr }}$ de funções $g_{\text {nrestr }}: \Re^{n} \rightarrow \Re$, que originam um subconjunto $F=\left\{\vec{x} \in M \mid g_{j}(\vec{x}) \geq 0 \forall j\right\} \subseteq M$, conhecido como conjunto de soluções viáveis de $f$.

Outras dificuldades podem surgir na otimização de problemas reais, como grande número de variáveis, elevado número de restrições, ruídos, não-linearidades, descontinuidades, objetivos conflitantes ou representados por funções complexas. De acordo com o problema abordado, muitas vezes um processo de otimização não é capaz de identificar um máximo global para o problema, mas o simples fato de se determinar uma solução viável melhor que uma já conhecida, pode ser considerado um grande resultado (Bäck, T. et alii, 1991).

\section{MINIMIZAÇÃO DE PERDAS}

Os materiais empregados nos condutores elétricos das redes de distribuição apresentam certa medida de oposição à corrente elétrica, ou seja a conhecida resistência elétrica. Diversos equipamentos instalados em redes elétricas são modelados com emprego deste conceito, para representar uma componente de seu funcionamento associado com perdas, isto é, a parcela de energia que aquele componente retira do sistema, devido ao seu funcionamento.

Com investimentos na rede existente, pode-se reduzir os custos associados com perdas nos condutores se diminuirmos a resistência elétrica em cada trecho, mantidas as situações de carregamento, com o emprego de condutores de maior seção transversal. Alternativamente, podem ser consideradas novas rotas, novos circuitos ou a instalação de novas subestações, como forma de aliviar o carregamento dos condutores existentes e, portanto, o valor de perdas.

Outra alternativa que permite a alteração da topologia da 
rede, em curto prazo, é considerar a mudança dos estados dos dispositivos de seccionamento da rede. Para aumentar a confiabilidade do sistema, freqüentemente são instaladas chaves entre diferentes circuitos, que são dispositivos normalmente abertos, existentes especificamente para o atendimento de regiões da rede sob condições especiais de contingência ou manutenção. Além destas chaves de interconexão entre circuitos, são também instalados dispositivos de proteção e seccionamento em cada circuito, que são elementos normalmente fechados, como chaves seccionadoras de linha, fusíveis e disjuntores.

Uma formulação para o problema de minimização de perdas, na ausência de investimentos na rede, considera os custos das perdas elétricas, $C_{\text {perdas }}$, associados com uma determinada configuração na forma:

$$
\min C_{\text {perdas }}=C_{p} \cdot \min \sum_{i j \in \Omega_{t}} r_{i j}\left(x_{j i}^{2}+x_{i j}^{2}\right)
$$

sujeito a:

$$
\begin{aligned}
& \sum_{j \in \Omega_{i}}\left(x_{j i}-x_{i j}\right)=D_{i}, \quad i \in \Omega_{b} \\
& x_{j i}+x_{i j} \leq x_{\text {max }, i j} \quad i j \in \Omega_{t} \\
& \Delta V_{j}-\Delta V_{i}+z_{i j}\left(x_{j i}-x_{i j}\right)=0, \quad i j \in \Omega_{t} \\
& \Delta V_{i} \leq \Delta V_{\max } \quad i \in \Omega_{b}
\end{aligned}
$$

onde:

$x_{i j}, x_{j i}$ fluxos de corrente do nó $i$ para $j$ e do nó $j$ para $i$, ambos positivos;

$r_{i j}$ resistência do nó $i$ para $j$.

$C_{p}$ custo unitário das perdas por unidade de demanda (por exemplo, em $\mathrm{R} \$ / \mathrm{kW}$ );

$D_{i}$ demanda do nó $i$;

$\Omega_{i}$ conjunto de nós conectados ou a serem conectados ao nó $i$;

$x_{\max , i j}$ limite de carregamento do trecho $i j$;

$\Delta V_{i}$ queda de tensão acumulada (da SE até o nó $i$ ), restrita a um valor limite $\Delta V_{\text {Max }}$

$\Omega_{b}$ e $\Omega_{t}$ conjuntos de barras e trechos da rede.

Uma possibilidade para simplificar o problema de otimização enunciado em (1) consiste em incluir suas restrições diretamente na função objetivo inicial, de maneira a degradar seu valor quando tais restrições não forem respeitadas em alguma configuração. Através do Método das Penalizações (Novaes, A. G., 1978), pode-se obter um problema equivalente, sem restrições, na forma:

$$
\min \left(C_{\text {perdas }}\right)+\sum_{j=1}^{n_{\text {restr }}} r_{j} P_{j}
$$

onde cada fator de penalização $P_{j}$ corresponde a uma das restrições $n_{\text {restr }}$ do problema original (1), ponderadas por fatores $r_{j}$. Nos problemas de minimização, em geral, são utilizados fatores $r_{j}$ elevados, o que afasta os algoritmos de otimização das soluções inviáveis.

\section{ALGORITMOS GENÉTICOS}

O Algoritmo Genético (Holland, J. H., 1975; De Jong, K., 1975) representa uma classe de algoritmos de otimização que empregam mecanismo de pesquisa probabilístico de soluções, baseado no processo de evolução biológico, combinando aspectos da mecânica da genética e da seleção natural de indivíduos. Apesar de envolver procedimentos aleatórios, os AGs se distanciam muito dos métodos de busca puramente aleatórios.

Conforme ressaltado em (Goldberg D. E., 1989), os AGs são métodos numéricos de otimização que, para maior robustez, se diferenciam dos outros em quatro aspectos fundamentais:

- Os AGs trabalham com codificação de parâmetros, ao invés dos pârametros originais do problema.

- Os AGs pesquisam soluções ótimas a partir de um conjunto de soluções, não a partir de uma única.

- O AG básico emprega uma função de avaliação para as diferentes soluções pesquisadas, codificadas em seqüências de comprimento conhecido, conhecidas como strings, empregando alfabeto binário na representação destas sequiências.

- Os AGs utilizam regras probabilísticas na pesquisa de novas soluções e não determinísticas.

\subsection{Codificação}

O AG codifica as soluções de um problema em strings, seqüências de bits de comprimento fixo, que guardam relação explícita com a formulação do problema. Esta representação guarda semelhanças com a forma sequiencial de organização de informações genéticas em cromossomos, onde cada alelo traz informações sobre determinadas características do 


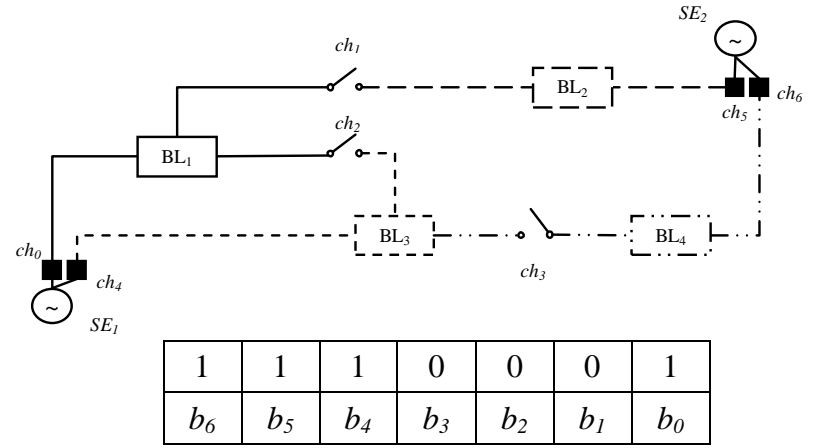

Figura 1: Exemplo de representação de um string (ou indivíduo)

indivíduo, conforme recebidas de seus antecessores. Devido a essa semelhança, os strings são freqüentemente chamados indivíduos.

O AG básico emprega alfabeto binário para composição dos indivíduos, onde um indivíduo " $i$ " qualquer, de dimensão " $l$ " é tal que $i \in B^{l}, B=\{0,1\}$. Esta codificação será comum aos problemas aqui analisados.

Conforme exposto em (Goldberg D. E., 1989), existem inúmeras maneiras de codificação do problema, ou de mapear soluções do problema original em strings de comprimento fixo. Entretanto, as particularidades de cada problema devem ser consideradas na codificação, evitando que os procedimentos aleatórios do algoritmo acabem gerando muitas soluções inviáveis, o que pode tornar a convergência difícil, senão impossível.

A Figura 1 apresenta uma codificação possível para o problema de configuração de redes, onde cada bit está associado com o estado da chave correspondente (se a chave $b_{i}$ está aberta o bit vale 0 , caso contrário, vale 1). Uma codificação mais interessante que a exibida na fig. 1 surge quando se considera o conceito de blocos de carga e famílias de blocos (Kagan, N., 1999). Blocos de carga compreendem conjuntos de barras do sistema, delimitados por chaves ou finais de linha. Codificando os indivíduos segundo as chaves incidentes em cada bloco e, notando que em redes radiais e conexas cada bloco se conecta ao restante do sistema fonte através de uma única chave, resulta que o número de chaves fechadas no sistema equivale ao seu número de blocos. Desta forma, é possível identificar, a priori, quais indivíduos devem ser eliminados e quais devem ser decodificados em uma rede válida e avaliados segundo os resultados de um fluxo de potência correspondente.

Nesta codificação, a partir da rede da Figura 1, tem-se o string representado na Figura 2.

\begin{tabular}{|c|c|c|c|c|c|}
\hline \multicolumn{2}{|c|}{ BL1 } & $B L 2$ & \multicolumn{2}{|c|}{ BL3 } & BLA \\
\hline 0 & 0 & 1 & 1 & 0 & 1 \\
\hline \multicolumn{2}{|c|}{$c h_{0}$} & $c h_{l}$ & \multicolumn{2}{|c|}{$c h_{2}$} & $c h_{3}$ \\
\hline \multicolumn{2}{|c|}{$c h_{I}$} & $c h_{5}$ & \multicolumn{2}{|c|}{$c h_{3}$} & $c h_{6}$ \\
\hline \multicolumn{2}{|c|}{$c h_{2}$} & & \multicolumn{2}{|c|}{ ch4 } & \\
\hline
\end{tabular}

Figura 2: Representação de um string na codificação proposta neste trabalho, representando o sistema da Figura 1.

\subsection{População Inicial}

O AG inicia gerando um número pré-definido de soluções iniciais, aleatoriamente, formando a população inicial. Computacionalmente, a implementação deste procedimento é muito simples, em função de existirem boas funções geradoras de números aleatórios, na maioria das ferramentas de programação. Este procedimento torna-se adequado à codificação dos strings segundo os blocos do circuito, conforme mencionado no item anterior.

\subsection{Função de Avaliação e Restrições}

Devido ao uso de alfabeto binário para representação dos alelos, o AG básico manipula diretamente apenas os problemas pseudo-booleanos, na forma $f: B^{l} \rightarrow R$, onde a função objetivo $f$ equivale à própria função de avaliação (Bäck, T., 1993).

Para o emprego do AG em problemas de otimização com restrições, como o de reconfiguração de redes, após definida a codificação dos strings, compõe-se uma função de avaliação para os indivíduos, a partir da função objetivo original do problema.

Para esta função de avaliação considerar as restrições originais do problema, frequientemente são empregados fatores de penalização, que tendem a piorar o valor da avaliação, quanto mais a solução proposta se afasta da situação ideal.

Para reconfiguração de redes, uma possibilidade seria definirmos uma função de avaliação, $f_{\text {aval }}$, proporcional a um valor mínimo de perdas perda $a_{\text {min }}$, com um número $n_{\text {restr }}$ de restrições, conforme o valor de perdas $_{i}$, de cada indivíduo $i$ (Kagan, N., 1999):

$$
f_{\text {aval }, i}=\frac{p e r d a_{\text {min }}}{\operatorname{perdas}_{i}+\sum_{j=1}^{n_{\text {rest }}} r_{j} P_{i, j}}
$$

As penalizações $P_{i, j}$ consideradas neste trabalho, ponderadas com fatores $r_{j}$, contabilizam o excesso de carregamento das subestações $\left(P_{S E i}\right)$, a sobrecarga em trechos dos circui- 
tos $\left(P_{T R i}\right)$ e a existência de cargas não atendidas $\left(P_{C i}\right)$ no sistema, conforme a rede representada pelo indivíduo $i$, da seguinte maneira:

$$
P_{S E i}=\sum_{n=0}^{n_{S E}} k_{S E n}
$$

onde $k_{S E n}=k_{S E d}$, se a $S E_{n}$ estiver desconectada do sistema;

e $k_{S E n}=k_{S E s}$, se $S E_{n}$ fornecer acima de seu nominal.

$$
P_{T R i}=\sum_{t=0}^{n_{T R}} k_{T R t}
$$

onde $k_{T R t}=k_{T R d}$, se o trecho $t$ estiver desconectado do sistema;

e $k_{T R t}=k_{T r s}$, se o fluxo no trecho $t$ estiver acima do nominal.

$$
P_{C i}=n_{c}
$$

onde $n_{c}$ corresponde ao número de cargas não atendidas.

Definidos os critérios de penalização, compõe-se o fator de penalização $P_{j}$ associado a cada indivíduo $i$, somando-se os valores de $P_{S E i}, P_{T R i}$ e $P_{C i}$ :

$$
P_{i, j}=P_{S E i}+P_{T R i}+P_{C i}
$$

Outros aspectos poderiam ser facilmente considerados pelo algoritmo, como minimização de energia não distribuída ou otimização dos perfis de tensões, por exemplo, bastando-se para tanto a alteração ou inserção de novas penalizações.

\subsection{Operador Genético Seleção}

O operador probabilístico Seleção Proporcional (Goldberg D. E., 1989) empregado no AG básico, sorteia os indivíduos de uma população para cruzamento, onde cada um tem chances de sorteio proporcionais aos seus respectivos valores de avaliação, isto é, a probabilidade de sorteio de um indivíduo $i$ é dado por $p_{i}=\Phi\left(a_{i}\right) / \sum_{j=1}^{u} \Phi\left(a_{j}\right)$, onde $\mu$ representa o tamanho da população e $\Phi: B^{l} \rightarrow \mathcal{R}^{+}$, a função de avaliação. Este método de seleção é também conhecido por "Regra da Roleta".

Especialmente em populações pequenas, ou no início da execução, é comum encontrarmos grandes diferenças nas avaliações dos indivíduos ou indivíduos excepcionalmente bem avaliados, quando a avaliação média da população é baixa. Mais ao final da execução, é freqüente termos a média da população próxima do melhor avaliado. Nestes casos, o AG poderá produzir populações com baixa variedade de indivíduos, resultando em indesejada convergência prematura, ou um mero "caminhar entre os medíocres".

Através do escalonamento (Goldberg D. E., 1989) a avaliação de cada indivíduo será recalculada como $f^{\prime}=a \cdot f+b$, onde $f^{\prime}$ representa a função de normalização das avaliações, $f$ é a função original de avaliação e os coeficientes $a$ e $b$ podem ser escolhidos de diferentes maneiras. Pela implementação dos demais operadores genéticos, torna-se necessário que as avaliações escalonadas não resultem inferiores a zero. Uma possibilidade, proposta em (Goldberg D. E., 1989) admite $f_{\text {med }}^{\prime}=f_{\text {med }}$ e $f_{\text {max }}=C_{\text {mult }} \cdot f_{\text {med }}^{\prime}$, com $f_{\text {min }}^{\prime}>0$ e $C_{\text {mult }}$ entre 1,2 e 2,0. Desta forma, os indivíduos melhor avaliados permanecem com maiores chances de sorteio mas os de avaliação inferior têm suas chances aumentadas. Isto auxilia a manter a diversidade de informações necessária na população, para que novas soluções sejam obtidas, a partir de recombinações com diferentes indivíduos.

O termo pressão seletiva ("selective pressure”) (Bäck, T., 1994) é frequentemente empregado para denotar quanto o método de seleção empregado considera o valor de avaliação dos indivíduos: quanto maior a "pressão" ou quanto mais o método de seleção considera a avaliação dos indivíduos, maior a tendência à exploração das soluções já existentes e maior a velocidade de convergência. Por outro lado, esquemas de seleção que pouco consideram o valor de avaliação das soluções tendem a pesquisar melhor o espaço de soluções, aumentando a confiabilidade da solução indicada como ótima.

Como alternativas ao método de seleção proporcional do AG básico, o Torneio ("Tourment") sorteia aleatoriamente dois indivíduos para cruzamento e seleciona para participar de um cruzamento aquele com melhor avaliação. Uma variante deste método, o Torneio-q ("q-Tourment") sorteia um número $q$ de indivíduos, $q>2$ e seleciona o melhor deles. Uma análise matemática e maiores detalhes sobre este operador podem ser consultados em (Bickle, T. and Lothar, T., 1995).

Outro operador de seleção que emprega implicitamente o valor de avaliação de cada solução é o Ranking Linear (Bäck, T. and Hoffmeister, F., 1991). Neste método, a população deve ser ordenada conforme os valores de avaliação de cada indivíduo, considerando-se apenas os $\mu$ melhor avaliados. Cada indivíduo recebe uma probabilidade idêntica de sorteio $1 / \mu$, independente do seu valor absoluto de avaliação. Uma forma de atribuir maior probabilidade aos melhores avaliados é considerada no Ranking Uniforme, onde a probabilidade $p_{i}$ de 
sorteio de cada indivíduo é ajustada através da expressão

$$
p_{i}\left(a_{i}^{g}\right)=1 / \lambda\left(\eta_{\max }-\left(\eta_{\max }-\eta_{\min }\right) \cdot(i-1) /(\lambda-1)\right)
$$

onde $\eta_{\min }=2-\eta_{\max }$ e $1 \leq \eta_{\max } \leq 2, a_{i}^{g}$ corresponde ao indivíduo $i$ e $\lambda$ é o número de indivíduos na população $g$ (Bäck, T. and Hoffmeister, F., 1991).

\subsection{Elitismo}

O AG básico descarta a geração anterior e considera para a futura apenas os descendentes obtidos. A técnica Elitista consiste em reintroduzir o indivíduo melhor avaliado de uma geração para a seguinte, evitando a perda de informações importantes presentes em indivíduos de alta avaliação e que podem ser perdidas durante os processos de seleção e cruzamento. Algumas técnicas controlam o número de vezes que o indivíduo pode ser reintroduzindo, o que contribui para evitar convergência a máximos locais.

Outra forma de implementar o elitismo seria ao final das operações de cruzamento e mutação, considerando para a geração futura os melhores entre os pais e descendentes. Esta técnica, naturalmente reintroduz os melhores avaliados na geração seguinte, indefinidamente. Em AGs, as gerações são mantidas com número fixo de indivíduos, $\mu$, que geram $\lambda$ descendentes, sendo $\mu=\lambda$. Este método de seleção é também conhecido como $(\mu+\lambda)$.

\subsection{Cruzamento}

O operador Cruzamento troca arbitrariamente sub-strings entre dois indivíduos selecionados para reprodução. O comprimento e posições dos sub-strings são determinados aleatoriamente mas são idênticos para ambos os indivíduos. Este operador recombina soluções e favorece a exploração do espaço de soluções em torno das já conhecidas e direciona o processo de evolução, de maneira geral. O AG básico considera que esta troca ocorrerá com determinada probabilidade $p_{c}\left(p_{c} \approx 0,6, p_{c} \in[0,75 ; 0,95]\right.$, ou $\left.p_{c} \approx 0,95\right)$ (Bäck, T., 1993a), isto é, existe a possibilidade da troca não ocorrer e ambos indivíduos resultarem idênticos na população seguinte.

O operador cruzamento do AG básico emprega apenas um ponto para troca de informações, sendo conhecido portanto como cruzamento em um ponto.

Os trabalhos (Spears, W. M. and De Jong, K. A., 1991) e (Spears, W. M. and De Jong, K. A., 1991a) apresentam formas de recombinar soluções, em dois ou $n$ pontos, analisando aspectos como a escolha do tamanho da população e dos operadores de recombinação, além de fornecer elementos para melhor compreender quando e como utilizar tais recursos. Empiricamente, o trabalho indica que existem situações onde a definição de um número maior de pontos de cruzamento é benéfico.

O Cruzamento uniforme (Syswerda, G., 1989) aqui abordado é um operador que compara, bit a bit, dois strings selecionados para reprodução. Caso os valores em determinado locus coincidam, ambos descendentes recebem este valor. Caso estes valores sejam diferentes, eles serão trocados com uma probabilidade fixa, $p_{c}$. Este método é teoricamente analisado em (Spears, W. M. and De Jong, K. A., 1991) onde se destaca a necessidade de aplicar os diferentes operadores de cruzamento na busca do equilíbrio entre a pesquisa entre as soluções conhecidas e a exploração do espaço de soluções.

Em termos práticos, destaca-se a versatilidade deste operador em conciliar estes aspectos através do ajuste de $p_{c}$.

Yang (Yang, S., 2002) propõe o uso de algumas informações implícitas ao longo da evolução, como a tendência dos alelos convergirem para um determinado valor, quando o algoritmo se encontra "distante" das gerações iniciais. Este método de cruzamento converte a freqüência de determinado alelo, $f_{1}(i, t)$ em uma probabilidade de troca, $p_{s}(i, t)$, com tendência a preservar os alelos mais freqüentes:

a) Ajuste "triangular":

$$
p_{S}(i, t)=P_{M a x}-2\left|f_{1}(i, t)-0,5\right|\left(P_{\text {Max }}-P_{\text {Min }}\right)
$$

b) Ajuste "trapezoidal":

$$
\begin{gathered}
p_{S}(i, t)=P_{\text {Max }}, \text { para }\left|f_{1}(i, t)-0,5\right| \leq a \\
p_{S}(i, t)=\frac{b-\left|f_{1}(i, t)-0,5\right|}{b-a}\left(P_{\text {Max }}-P_{\text {Min }}\right)+P_{\text {Min }}, \\
\text { para } a<\left|f_{1}(i, t)-0,5\right|<b \\
p_{S}(i, t)=P_{\text {Min }}, \text { para }\left|f_{1}(i, t)-0,5\right| \geq b \quad \text { (10) }
\end{gathered}
$$

c) Ajuste "exponencial":

$$
p_{S}(i, t)=\alpha e^{\left(-\left(f_{1}(i, t)-0,5\right)^{2} / \beta\right)}
$$

\subsection{Mutação}

No AG básico, o papel central da pesquisa por novas soluções se baseia na seleção e reprodução dos indivíduos.

O operador mutação nos AGs freqüentemente encontra três argumentos. O primeiro atribui ao operador um papel meramente secundário, servindo apenas como paleativo para evitar a perda permanente de alelos. Outro, afirma que o operador deve manter uma taxa constante e baixa, ao longo da 
execução do algoritmo e, finalmente, que o operador mutação deve empregar uma taxa única, para todos genes de todos indivíduos, em determinada população.

Algoritmos Evolutivos podem codificar uma probabilidade de mutação no indivíduo, implementando um valor diferente para os indivíduos, a cada geração. Inicialmente, admite-se valores altos para a probabilidade de mutação, para favorecer a exploração do espaço de soluções a partir das iniciais, obtidas aleatoriamente. Ao longo da execução, espera-se que o valor de mutação tenda a valores muito pequenos e que o algoritmo estabilize os indivíduos ao redor do valor ótimo, o que também contribui para sinalizar a convergência do algoritmo.

Ochoa et alii (1999) argumentam que não há necessidade de se alterar o valor da probabilidade de mutação, quando empregada recombinação ou cruzamento, pois a recombinação também auxilia na exploração do espaço de soluções, sugerindo o uso de recombinação nas Estratégias Evolutivas. Thierens (2002) avalia uma série de alternativas para o operador nos AGs, na ausência do operador cruzamento, indicando como trabalho futuro a determinação de uma forma de decaimento para a probabilidade de mutação. Como forma de reforçar a exploração, ao início da execução, este trabalho irá avaliar o ajuste desta probabilidade conforme:

$p_{m}(g)=P_{m, \min }+\left(P_{m, \max }-P_{m, \min }\right) \cdot e^{\left(1 / G_{n} \cdot \ln \left(P_{m, m i n}\right) \cdot g\right)}$

onde $P_{m, \min }$ é o valor aproximado da probabilidade de mutação a partir da geração $G_{n}$ e $P_{m, \max }$ é o valor inicial de $p_{m}$.

\subsection{Critério de convergência}

Foram utilizados dois critérios de convergência:

(a) $\mathrm{O}$ primeiro analisa o melhor indivíduo (incumbente). $\mathrm{Se}$ este não tem uma melhoria na avaliação do melhor indivíduo durante um número fixo de gerações $\left(N_{g e r}\right)$, é considerado que o processo convergiu. Caso contrário adota-se o segundo critério;

(b) O segundo é fixar um número máximo de gerações $\left(N_{\max }\right)$, onde $\left(N_{\text {ger }}<N_{\max }\right)$. Este critério levará o processo a continuar, até atingir a melhor indivíduo.

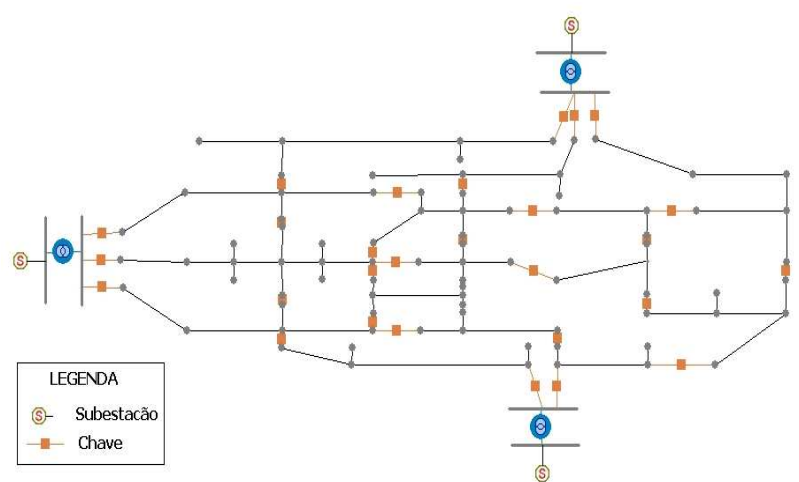

Figura 3: Sistema de distribuição para minimização de perdas

\section{RESULTADOS}

\subsection{Resultados com rede hipotética}

A metodologia descrita foi aplicada inicialmente para a resolução de um problema de minimização de perdas em uma rede de distribuição (Kagan, N. 1999), com 89 barras, 20 chaves e três subestações, conforme ilustrado na Figura 3. Nesta rede, é conhecida a configuração ótima, gerada a partir de um método de busca exaustiva (Schmidt, H. P. et alii, 2005).

Para avaliação e comparação do desempenho dos diferentes operadores propostos, a rede proposta foi otimizada cinco vezes com cada combinação de operadores genéticos, conforme Tabela 1 .

Tabela 1: Combinação de Operadores Analisados

\begin{tabular}{|c|c|c|c|}
\hline \multirow{7}{*}{ Seleção } & \multicolumn{3}{|c|}{ Proporcional (ou "Roleta") } \\
\hline & \multirow{2}{*}{ Torneio } & $q=4$ & \\
\hline & & $q=8$ & \\
\hline & \multirow{4}{*}{ Ranking } & Linear & $\mu=4$ \\
\hline & & \multirow{3}{*}{ Uniforme } & $\mu=4 ; \eta_{\operatorname{Max}}=1,3$ \\
\hline & & & $\mu=8 ; \eta_{\operatorname{Max}}=1,3$ \\
\hline & & & $\mu=8 ; \eta_{\operatorname{Max}}=1,6$ \\
\hline \multirow{4}{*}{ Cruzamento } & \multicolumn{3}{|l|}{ Um ponto } \\
\hline & \multicolumn{3}{|c|}{ Dois pontos } \\
\hline & \multirow{2}{*}{ Uniforme } & \multicolumn{2}{|l|}{$p_{c}=0,65$} \\
\hline & & \multicolumn{2}{|l|}{$p_{c}=0,85$} \\
\hline
\end{tabular}

Cada combinação entre operadores de seleção e cruzamento foi executada 5 vezes, em geração de 12 indivíduos, limitada a 500 gerações ou 120 consecutivas sem melhoria na avali- 


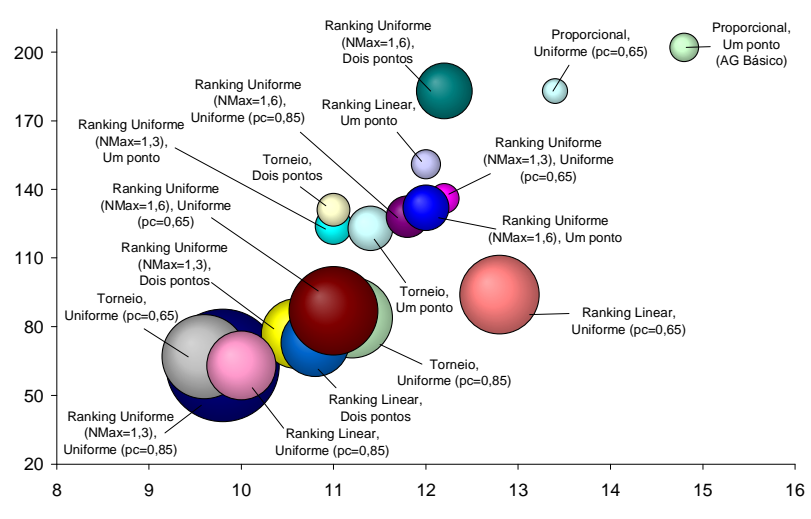

Figura 4: Avaliação dos métodos de seleção e cruzamento

ação do melhor indivíduo, considerando apenas a execução em curso. A probabilidade de mutação foi mantida fixa, em 0,10 .

O gráfico exibido pela Figura 4 exibe de maneira qualitativa os resultados obtidos. No eixo das abcissas, representa-se o tempo médio de processamento das cinco execuções, em um Pentium III 650MHz, considerando-se os limites mencionados. Nas ordenadas, é indicada a maior geração (seqüencial) onde se obteve a resposta do problema e o tamanho de cada bolha equivale ao inverso do desvio padrão da geração onde se obteve a resposta do problema. Procura-se aqui, através da rede do exemplo, identificar qual combinação tenderia a obter a melhor solução em menor tempo, consistentemente. Desta forma, as bolhas maiores e mais próximas da origem correspondem aos melhores parâmetros.

O gráfico da Figura 4 não exibe os resultados dos operadores Seleção Torneio $(q=8)$ e Proporcional, com Cruzamento em Dois Pontos e Uniforme $\left(p_{c}=0,65\right)$ por não terem convergido à melhor resposta em todas as execuções. Este resultado já era esperado pois, para populações com poucos indivíduos, como são os casos analisados, o valor de $q=8$ provoca uma alta pressão seletiva.

A partir do gráfico da Figura 4, pode-se notar que o AG básico teve o pior desempenho, em todos os aspectos avaliados: número de gerações para convergência, desvio padrão desse número, valor da solução indicada e tempo de processamento.

Particularmente a escolha Ranking Uniforme, com $\mu=4$ e $\eta_{\text {Max }}=1,3$, e Cruzamento Uniforme com $p_{c}=0,85$ resultou em convergência média na geração 39 (mínima 32 e máxima 64), com menor desvio padrão, o que pode indicar que o método poderia trazer a solução ótima em menor número de gerações, consistentemente. Eventualmente, de acordo com as características do sistema analisado, os parâmetros seriam ligeiramente alterados mas este resultado indica que não se deve considerar muitos indivíduos da população, nem aumentar demasiadamente a ponderação $\eta_{M a x}$, o que poderia resultar em indesejada convergência a máximos locais. Com relação ao operador Cruzamento Uniforme, a tendência seria manter a probabilidade de troca em valores mais altos, o que força o algoritmo a pesquisar melhor o espaço de soluções.

O operador seleção Torneio $(q=4)$, associado ao operador Cruzamento Uniforme, com probabilidade de troca menor $(0,65)$, obteve convergência, em média, na geração 37 (mínima na 21 e máxima na 67), outro resultado notável. Nos testes feitos para operadores com o parâmetro $q$ menor $(q<4)$, obteve-se a convergência para a mesma configuração de rede, porém o número de gerações necessário para convergência aumentou.

O Torneio é o operador de seleção mais simples de ser implementado, resumindo-se ao sorteio aleatório de $q$ indivíduos da população, mas ainda assim, capaz de fornecer bons resultados. Neste caso, os tempos de processamento tendem a ser menores, pois não existe a necessidade de ordenar a população (Ranking) ou escalonar as avaliações (Proporcional).

Em seguida, foram avaliados os operadores Cuzamento uniforme estatístico e Mutação com probabilidade variável, conforme Tabela 2, empregando-se seleção com ranking uniforme, $\mu=4$ e $\eta_{M a x}=1,3$, identificado a partir da Figura 4 como uma boa alternativa de seleção. Os demais parâmetros permanecem inalterados.

Os resultados destas simulações são exibidos na Figura 5, a seguir, onde também é exibido um resultado da Figura 4, para efeito de comparação.

A rede ótima resultante, em todas as execuções mencionadas nas Figuras 4 e 5, é exibida na Figura 6. Destaca-se que estes mesmos resultados foram obtidos utilizando os algoritmos propostos em (Guerra, W. et alii, 2006), (Cebrian, J. et alii, 2003) e (Schmidt, H. P. et alii, 2005). Comparando o esforço computacional com outro método formulado por AG, o método apresentado em (Cebrian, J. et alii 2003) obteve uma convergência, em média, na geração 47 , quando considerando o mesmo número de indivíduos da população, ou seja, um valor um pouco superior ao método aqui analisado.

\subsection{Resultados com rede real}

Para validação dos resultados obtidos no item anterior, foi considerado o problema de minimização de perdas de uma rede real, exibida pela Figura 7.

As principais características da rede ilustrada pela Figura 7 são resumidas na Tabela 3 .

De acordo com os resultados obtidos no item 5.1, 
Tabela 2: Operadores Genéticos

\begin{tabular}{|c|c|c|}
\hline \multirow{5}{*}{$\begin{array}{l}\text { Uniforme } \\
\text { Estatístico }\end{array}$} & Ajuste Triangular & $\begin{array}{l}P_{\text {Max }}=0,85 \\
P_{\text {Min }}=0,35\end{array}$ \\
\hline & Ajuste Trapezoidal & $\begin{array}{l}P_{\text {Max }}=0,85 \\
P_{\text {Min }}=0,35 \\
A=0,10 ; b=0,40\end{array}$ \\
\hline & & $P_{\text {Max }}=0,85$ \\
\hline & & $\begin{array}{l}P_{\text {Min }}=0,35 \\
A=0,20 ; b=0,30\end{array}$ \\
\hline & Ajuste Exponencial & $\begin{array}{l}\alpha=0,85 ; \beta=0,03 \\
\alpha=0,85 ; \beta=0,01 \\
\alpha=0,65 ; \beta=0,03 \\
\alpha=0,65 ; \beta=0,01\end{array}$ \\
\hline $\begin{array}{l}\text { Mutação } \\
\text { Variável }\end{array}$ & \multicolumn{2}{|c|}{$G_{n}=200 ; P_{M a x}=0,30 ; P_{\text {Min }}=0,05$} \\
\hline
\end{tabular}

selecionam-se três alternativas de operadores de seleção: Torneio, Ranking Uniforme e Linear. Estas alternativas são combinadas com o operador Cruzamento Uniforme, para avaliação de desempenho na rede mencionada. Considerase, igualmente, os operadores e parâmetros referentes ao AG Básico, para permitir a comparação dos resultados obtidos. A Tabela 4 relaciona os operadores empregados para a otimização dessa rede real e a Tabela 5 complementa as informações dos demais parâmetros empregados.

Para o estudo desses casos, foi empregado o ambiente de Planejamento de Redes de Distribuição Interplan (Kagan, N. et alii, 2004) que permite a obtenção de informações da rede diretamente do sistema corporativo de empresas distribuidoras. A partir das informações de faturamento de ener-

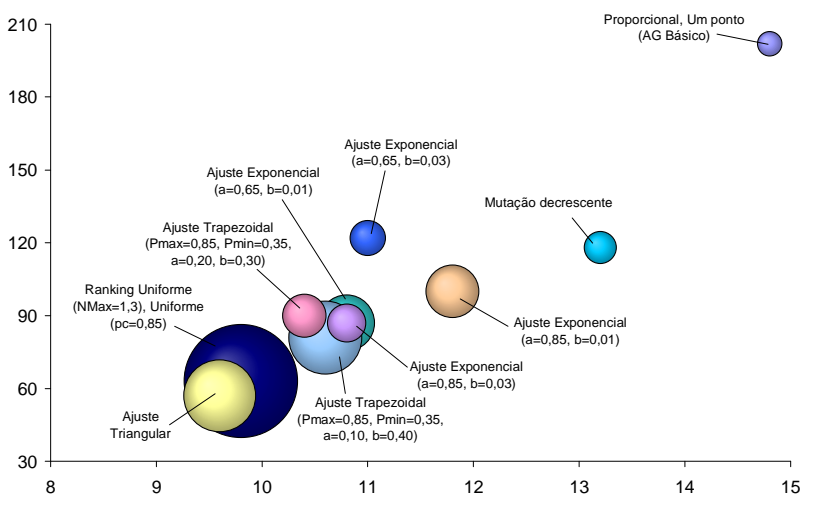

Figura 5: Avaliação dos métodos de cruzamento uniforme estatístico e mutação variável.

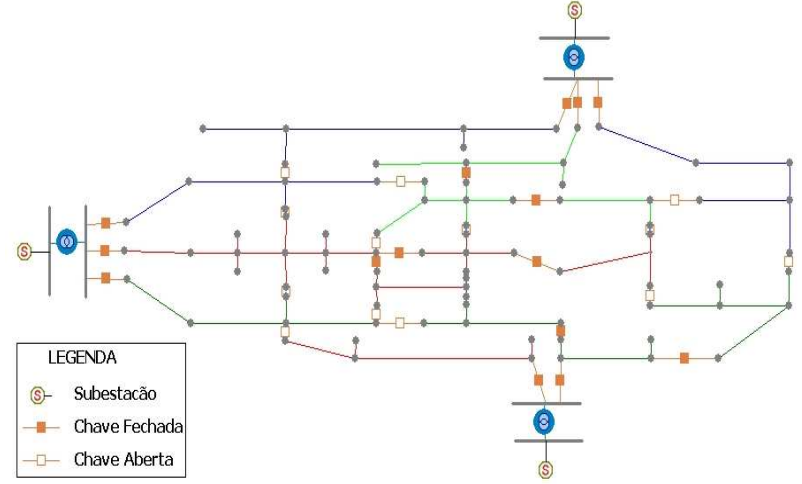

Figura 6: Rede ótima, considerando-se o objetivo único de minimização de perdas elétricas.

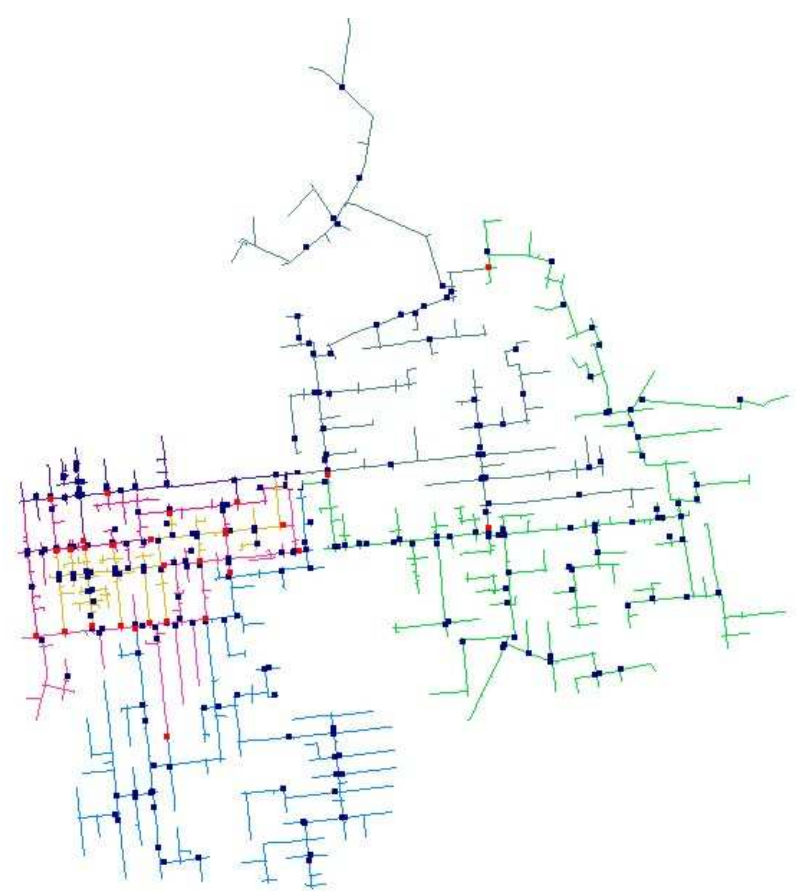

Figura 7: Representação da topologia da rede em estudo.

gia, estimam-se as demandas de cada centro de carga do sistema, através de curvas de carga típicas para cada categoria de consumidor, obtidas estatisticamente. $\mathrm{O}$ valor de perdas em energia é computado considerando-se quatro patamares de carregamento, ao longo do dia. Foi admitido que as configurações de rede em cada bloco permanecem inalteradas ao longo do estudo.

A partir de estudos de fluxo de potência, executados com esta ferramenta, os resultados de perdas na configuração original foram obtidos e são ilustrados pela Tabela 6 .

Os principais resultados obtidos nas quatro simulações, com 
Tabela 3: Resumo das principais características da rede elétrica da Figura 7

\begin{tabular}{|l|l|}
\hline Característica & Valor \\
\hline Subestações, total & 1 \\
\hline Número de circuitos & 7 \\
\hline Número de barras, total & 2400 \\
\hline Número de barras, com carga & 746 \\
\hline Potência instalada total (kVA) & $53.737,5$ \\
\hline $\begin{array}{l}\text { Número de dispositivos de seccio- } \\
\text { namento }\end{array}$ & 301 \\
\hline Número de ligações & 2130 \\
\hline
\end{tabular}

Tabela 4: Operadores empregados para otimização de uma rede real

\begin{tabular}{|l|l|l|}
\hline & Operador \\
\hline Caso & Seleção & Cruzamento \\
\hline $1-$ AG Básico & Proporcional & Um ponto \\
\hline 2 - Torneio & Torneio $(q=4)$ & $\begin{array}{l}\text { Uniforme } \\
\left(p_{c}=0,65\right)\end{array}$ \\
\hline $\begin{array}{l}3 \text { - Ranking } \\
\text { Linear }\end{array}$ & $\begin{array}{l}\text { Uniforme } \\
\left(\eta_{\text {Max }}=1,0\right)\end{array}$ & $\begin{array}{l}\text { Uniforme } \\
\left(p_{c}=0,85\right)\end{array}$ \\
\hline $\begin{array}{l}4-\text { Ranking } \\
\text { Uniforme }\end{array}$ & $\begin{array}{l}\text { Uniforme } \\
\left(\eta_{\text {Max }}=1,3\right)\end{array}$ & $\begin{array}{l}\text { Uniforme } \\
\left(p_{c}=0,85\right)\end{array}$ \\
\hline
\end{tabular}

operadores apresentados na Tabela 4, são exibidos na Tabela 7

Para avaliação da qualidade das respostas obtidas anteriormente, pelo fato de não ser conhecido o valor mínimo de perdas da rede analisada, outra execução foi efetuada, empregando-se o método 4 da Tabela 4, com 50 indivíduos

Tabela 5: Demais parâmetros empregados

\begin{tabular}{|c|c|}
\hline Parâmetro & Valor \\
\hline Número de execuções & 1 \\
\hline Número de gerações & 30 \\
\hline $\begin{array}{c}\text { Número de indivíduos } \\
\text { em uma população }\end{array}$ & 20 \\
\hline Prob. mutação & $1 \%$ \\
\hline Prob. cruzamento & $85 \%$ \\
\hline Custo de perdas & $40 \mathrm{R} \$ / \mathrm{MWh}$ \\
\hline
\end{tabular}

Tabela 6: Níveis originais de perdas na rede real, por alimentador

\begin{tabular}{|c|c|c|c|}
\hline Circuito & $\mathrm{kWh} / \mathrm{mês}$ & $\mathrm{MWh} / \mathrm{ano}$ & $\mathrm{R} \$ / \mathrm{ano}$ \\
\hline 1 & 3.755 & 45,06 & $1.802,40$ \\
\hline 2 & 47.431 & 569,172 & $22.766,88$ \\
\hline 3 & 17.970 & 215,64 & $8.625,60$ \\
\hline 4 & 4.611 & 55,332 & $2.213,28$ \\
\hline 5 & 6.231 & 74,772 & $2.990,88$ \\
\hline 6 & 4.646 & 55,752 & $2.230,08$ \\
\hline 7 & 986 & 11,832 & 473,28 \\
\hline Somatório: & 85.630 & $1.027,56$ & $41.102,40$ \\
\hline
\end{tabular}

Tabela 7: Ganhos obtidos na otimização de rede real com AG

\begin{tabular}{|c|c|c|c|c|}
\hline & & \multicolumn{2}{|c|}{$\begin{array}{l}\text { Ganho em relação à } \\
\text { configuração existente }\end{array}$} & $\begin{array}{l}\text { Ganho } \\
\text { em } \\
\text { relação } \\
\text { ao AG }\end{array}$ \\
\hline Caso & $\begin{array}{c}\text { Custo das } \\
\text { perdas, } \\
\text { anual }(\mathrm{R} \$)\end{array}$ & $(\mathrm{R} \$)$ & $(\%)$ & $(\%)$ \\
\hline $\begin{array}{c}\text { Configuração } \\
\text { de rede } \\
\text { existente }\end{array}$ & $41.102,40$ & - & - & - \\
\hline $\begin{array}{l}1 \text { - AG } \\
\text { Básico }\end{array}$ & $14.126,50$ & $26.975,90$ & $65,63 \%$ & - \\
\hline 2 - Torneio & $12.151,85$ & $28.950,55$ & $70,44 \%$ & $13,98 \%$ \\
\hline $\begin{array}{c}3 \text { - Ranking } \\
\text { Linear }\end{array}$ & $10.265,14$ & $30.837,26$ & $75,03 \%$ & $27,33 \%$ \\
\hline $\begin{array}{c}4 \text { - Ranking } \\
\text { Uniforme }\end{array}$ & $10.205,14$ & $30.897,26$ & $75,17 \%$ & $27,76 \%$ \\
\hline
\end{tabular}

em cada geração, em 50 gerações. Espera-se, desta forma, melhorar a pesquisa do espaço de soluções e aumentar as chances de se identificar uma resposta que se aproxime ainda mais da rede de mínimas perdas.

Neste caso, a melhor resposta foi obtida na Geração 45, correspondendo a um custo anual de perdas elétricas de $\mathrm{R} \$ 9.072,50$, o que representa cerca de $11 \%$ de melhoria sobre a melhor resposta obtida anteriormente, conforme relacionada na Tabela 7. Entretanto, convém ressaltar que os resultados apresentados nesta Tabela foram alcançados em cerca de oito minutos, em um computador com processador $2,4 \mathrm{GHz}$, enquanto o melhor resultado para este caso foi alcançado em aproximadamente meia hora. 


\section{CONCLUSÕES}

A partir das diferentes combinações de operadores e parâmetros analisados, este trabalho indica, qualitativamente, que o operador seleção Ranking teve o melhor desempenho, juntamente ao operador Cruzamento uniforme.

Das alternativas de Cruzamento uniforme com estatística, nota-se a partir do exemplo que emprega ajuste triangular, que a técnica pode melhorar o desempenho do AG e deve ser melhor estudada. Entretanto, esta traz um inconveniente ao usuário, de ter que identificar quando iniciar as estatísticas, informando um número de geração ou um valor de avaliação médio da população, o que nem sempre é prático ou conhecido de antemão.

Em todas as diferentes alternativas analisadas, notou-se que a seleção elitista melhorou o desempenho do AG em todos os aspectos, auxiliando na obtenção da solução ótima do problema, em menor número de gerações.

Conforme indicado em (Ochoa, G. et alii, 1999), a variação da probabilidade de mutação ao longo da execução não melhorou o desempenho do algoritmo. Em termos práticos, a probabilidade de mutação muito alta, mesmo nas gerações iniciais, tende a deixar o algoritmo instável, o que prejudica seu desempenho, de maneira geral.

O breve estudo sobre uma rede real, abordado no item 5.2, permitiu validar os resultados obtidos no item 5.1, com a rede hipotética, além de trazer outras indicações.

Conforme os resultados exibidos pela Tabela 7 , houve melhoria média superior a $70 \%$ no custo das perdas em relação à configuração existente, o que contribui para ilustrar o grande potencial da metodologia empregada. Variantes para os operadores genéticos canônicos foram capazes de melhorar a resposta do AG em mais de $27 \%$, o que permite afirmar que essas alternativas são capazes de melhorar a pesquisa do espaço de soluções viáveis e identificar melhores soluções.

Quando aplicado ao estudo de minimização de perdas de uma rede real, o AG Básico foi capaz de encontrar uma solução que representaria uma economia de mais de $65 \%$. Com emprego das variantes seleção Ranking Uniforme e Cruzamento Uniforme, identificou-se outra solução que equivaleria a mais de $75 \%$ de redução de custo mensal, mantidos os demais parâmetros de execução.

Conforme indicação dos resultados com a rede hipotética, o método a seleção Torneio obteve bom desempenho, identificando uma solução que equivaleria a cerca de $70 \%$ de economia em relação à configuração de rede existente, valor próximo do atingido com o método de seleção Ranking Uniforme, mas ainda inferior a este. No método da seleção por Torneio para redes pequenas, como a rede hipotética estudada, a variação deste operador pela redução do parâmetro $q$ (valores $q<4$ ) não influenciou o resultado, apesar do número de gerações para convergência ter sido aumentado.

Para a rede real estudada, uma simulação prolongada, contando com 50 gerações e 50 indivíduos e operadores que obtiveram melhor desempenho neste estudo, foi possível identificar uma configuração que reduz em $11 \%$ o melhor valor de perdas energéticas encontrado anteriormente. Isto significa dizer que as soluções representadas, embora tenham o valor de perdas reduzido, em todos os casos, correspondem a pontos sub-ótimos, ou situações próximas da melhor configuração, em termos de perdas elétricas.

Evidente que quaisquer configurações aqui obtidas não poderiam ser imediatamente aplicadas à rede real, dado que a formulação aqui empregada não considerou outros objetivos importantes, como o perfil de tensões e a divisão de carregamentos entre subestações e circuitos. Nem mesmo se deve considerar a ordem de grandeza dos ganhos alcançados nesses estudos como representativa das demais redes encontradas na prática.

É importante notar que a codificação do problema é essencial ao bom desempenho do AG. Nota-se que simplesmente não há convergência para o caso onde cada chave é associada a um bit do string, como ilustrado na Figura 1. Todos os exemplos ilustrados tiveram codificação conforme proposto em (Kagan, N., 1999), orientada aos blocos de carga do circuito.

\section{REFERÊNCIAS}

Bäck, T., Hoffmeister, F. and Schwefel, H. P. (1991). "A Survey of Evolution Strategies". Proceedings of the $4^{\text {th }}$ International Conference on Genetic Algorithms, San Diego, CA, In Morgan Kaufmann Publishers (Ed.) pp. 29 ,.

Bäck, T. (1993). "Optimal mutation rates in Genetic search", Proceedings of the $5^{\text {th }}$ International Conference on Genetic Algorithms, San Mateo, CA, In Morgan Kaufmann Publishers (Ed.), pp. 2-8.

Bäck, T. (1994). "Selective Pressure in Evolutionary Algorithms: a characterization of selection mechanisms”, Relatório Técnico, Dept. of Computer Science, University of Dortmund, Alemanha.

Bickle, T. and Lothar, T. (1995). "A mathematical analysis of Tournment selection", Proceedings of the Sixth International Conference on Genetic Algorithms, In Morgan Kaufmann Publishers (Ed.), San Francisco, CA, pp. $9-16$ 
Bäck, T., Hoffmeister, F. (1991). “Extended Selection Mechanisms in Genetic Algorithms”, Relatório Técnico, Dept. of Computer Science, University of Dortmund, Alemanha.

Bäck, T. (1993a). "Optimal mutation rates in Genetic search", Proceedings of the $5^{\text {th }}$ International Conference on Genetic Algorithms, San Mateo, CA. In Morgan Kaufmann Publishers (Ed.), USA, pp. 2 a 8.

Cebrian J.C.; Romero R.; Mantovani, J.R.S, (2005) “Algoritmos evolutivos dedicados a reconfiguração de redes radiais de distribuição sob demandas fixas e variáveis estudo dos operadores genéticos e parâmetros de controle", SBA. Sociedade Brasileira de Automática, Unicamp, v. 16, n. 03, p. 303-317.

De Jong, K. (1975). “An analysis of the behavior of a class of genetic adaptive systems". Tese de Doutoramento, University of Michigan, USA.

Enacheanu, B.; Raison, B.; Caire, R.; Devaux, O.; Bienia, W.; HadjSaid, N. (2008). "Radial Network Reconfiguration Using Genetic Algorithm Based on the Matroid Theory”, IEEE Transactions on Power Systems, Volume 23, Issue 1, pp 186 - 195, Feb.

Goldberg D. E. (1989). "Genetic Algorithms in Search, Optimization and Machine Learning". In Addison-Wesley (Ed.), Reading, MA.

Guerra, W. e Romero R. (2006) "Reconfiguração de sistemas de distribuição de energia elétrica utilizando a metaheurística busca em vizinhança variável" ,XVI Congresso Brasileiro de Automática, Campinas, v. 1. p. 1411-1416. Agosto.

Holland, J. H. (1975). “Adaptation in natural and artifcial systems”. The University of Michigan Press, Ann Arbour.

Kagan, N., Oliveira, C. C. B., Guaraldo, J. C., El Hage, F. S., Mèffe, A., Filho, M. M. (2004). "INTERPLAN - Uma ferramenta para o Planejamento Integrado de Sistemas de Distribuição de Alta, Média e Baixa Tensão”, 2004 IEEE T\&D Latin América, São Paulo.

Kagan, N. (1999). “Configuração de Redes de Distribuição através de Algoritmos Genéticos e Tomada de Decisão Fuzzy”. Tese de Livre Docência, Escola Politécnica da Universidade de São Paulo, SP.

Miranda, V.; Ranito, J. V; Proença, L. M. (1994). "Genetic algorithms in optimal multi-stage distribution network planning”, IEEE Transactions on Power Systems, vol.9, n. 4, Nov.
Nara, K.; Shiose, A.; Kitagawa, M.; Ishihara, T. (1992) "Implementation of genetic algorithm for distribution systems loss minimum re-configuration”, IEEE Trans. Power Syst., vol. 7, no. 3, pp. 1044-1051, Aug.

Novaes, A. G. (1978). "Métodos de Otimização: Aplicações aos Transportes”, Edgar Blücher Ltda., São Paulo, SP.

Ochoa, G., Harvey, I., Buxton, H. (1999). "On recombination and optimal mutation rates", Proceedings of the Genetic and Evolutionary Computation Conference, Vol. 1, In Morgan Kaufmann Publishers (Ed.), pp. 488495.

Spears, W. M. and De Jong, K. A. (1991). "On the Virtues of Parameterized Uniform Crossover", Proc. Fourth International Conference on Genetic Algorithms, In Morgan Kaufman Publishers (Ed.), pp. 230-236.

Spears, W. M. and De Jong, K. A. (1991a). “An analysis of multi-point crossover”, Foundations of Genetic Algorithms, In Morgan Kaufmann Publishers (Ed.), pp. 301-315.

Syswerda, G. (1989). "Uniform Crossover in Genetic Algorithms", Proc. Third International Conference on Genetic Algorithms, San Mateo (CA), In Morgan Kaufmann Publishers (Ed.).

Schmidt, H. P., Ida, N., Kagan, N., Guaraldo, J. C. (2005). "Fast Reconfiguration of Distribution Systems Considering Loss Minimization", IEEE Trans. Power Del. vol. 20, no. 3, pp. 1311-1319, August

Thierens, D. (2002). “Adaptative mutation rate control schemes in genetic algorithms", Relatório Técnico, Utrecht University, the Netherlands.

Yang, S. (2002). "Adaptive Crossover in Genetic Algorithms Using Statistics Mechanism”, Proc. of the Genetic and Evolutionary Computation Conference, In Morgan Kaufmann Publishers (Ed.), pp 650-657

\section{AGRADECIMENTOS}

OS AUTORES DESEJAM AGRADECER AOS DOUTORANDOS WILINGTHON GUERRA E JUAN CARLOS CEBRIAN AMASIFEN PELAS IMPORTANTES CONTRIBUIÇÕES E APOIO NA COMPARAÇÃO COM MÉTODOS BASEADOS EM METAHEURÍSTICAS.

\section{APÊNDICE}

Neste apêndice são apresentados nas tabelas A.1, A.2 e A.3 os dados para a rede hipotética do item 5.1. 
Tabela A.1 - Dados de cabos

\begin{tabular}{|c|c|c|c|c|}
\hline \multirow{2}{*}{ Índice } & \multirow{2}{*}{ Descrição } & \multicolumn{2}{|c|}{$\begin{array}{l}\text { Impedância } \\
\text { sequiência } \\
(\Omega / \text { de })\end{array}$} & $\begin{array}{c}\text { Corrente } \\
\text { direta } \\
\text { Admis- } \\
\text { sível } \\
(\mathbf{A})\end{array}$ \\
\cline { 3 - 4 } & & Resistência & Reatância & \\
\hline 1 & 4 CAA & 1.5900 & 0.5200 & 140. \\
\hline 2 & 4 CA & 1.5200 & 1.4800 & 125. \\
\hline 3 & $1 / 0 \mathrm{CA}$ & 0.6000 & 0.4500 & 215. \\
\hline 4 & 2/0 CA & 0.4700 & 0.4400 & 260. \\
\hline 5 & 4/0 CA & 0.3000 & 0.4200 & 340. \\
\hline 6 & $336 \mathrm{CA}$ & 0.1900 & 0.4000 & 510. \\
\hline 19 & $1 / 0 \mathrm{CAA}$ & 0.7000 & 0.5200 & 230. \\
\hline 23 & $336 \mathrm{CAA}$ & 0.1900 & 0.3800 & 530. \\
\hline
\end{tabular}

Tabela A.2 - Dados de Barras do Sistema da Fig. 3

\begin{tabular}{|c|c||c|c||c|c|}
\hline Barra & $\begin{array}{c}\text { Demanda } \\
(\mathbf{k W})\end{array}$ & Barra & $\begin{array}{c}\text { Demanda } \\
(\mathbf{k W})\end{array}$ & Barra & $\begin{array}{c}\text { Demanda } \\
(\mathbf{k W})\end{array}$ \\
\hline 54 & 2000.0 & 29 & 600.0 & 84 & 1000.0 \\
\hline 55 & 2000.0 & 7 & 500.0 & 85 & 400.0 \\
\hline 72 & 1000.0 & 12 & 800.0 & 27 & 100.0 \\
\hline 6 & 800.0 & 13 & 800.0 & 37 & 500.0 \\
\hline 8 & 2000.0 & 58 & 1000.0 & 25 & 100.0 \\
\hline 57 & 1000.0 & 59 & 600.0 & 46 & 500.0 \\
\hline 62 & 800.0 & 63 & 1000.0 & 31 & 500.0 \\
\hline 73 & 800.0 & 75 & 2000.0 & 42 & 100.0 \\
\hline 74 & 1000.0 & 76 & 500.0 & 48 & 500.0 \\
\hline 78 & 1000.0 & 79 & 800.0 & 50 & 500.0 \\
\hline 80 & 300.0 & 81 & 1500.0 & 47 & 500.0 \\
\hline 65 & 2000.0 & 33 & 500.0 & 49 & 500.0 \\
\hline 82 & 1000.0 & 9 & 100.0 & 51 & 300.0 \\
\hline 16 & 300.0 & 10 & 100.0 & 52 & 500.0 \\
\hline 69 & 3000.0 & 11 & 300.0 & & \\
\hline 83 & 700.0 & 17 & 500.0 & & \\
\hline 86 & 400.0 & 18 & 500.0 & & \\
\hline 32 & 500.0 & 20 & 800.0 & & \\
\hline 21 & 100.0 & 60 & 1000.0 & & \\
\hline 22 & 100.0 & 61 & 500.0 & & \\
\hline 30 & 500.0 & 39 & 500.0 & & \\
\hline & & & & & \\
\hline
\end{tabular}

Tabela A.3a - Dados de Ligações do Sistema da Fig. 3 (* - tipos de ligações: 0 -trecho sem chave, 1-trecho c/ chave NF, 2-trecho c/ chave NA)

\begin{tabular}{|c|c|c|c|c|}
\hline \multicolumn{2}{|l|}{$\begin{array}{l}\text { Nós } \\
\text { terminais }\end{array}$} & Cabo & $\begin{array}{c}\text { Comprim. } \\
(\mathrm{m})\end{array}$ & $\begin{array}{c}\text { Tipo } \\
(*)\end{array}$ \\
\hline 2 & 4 & 23 & 2000.0 & 0 \\
\hline 3 & 6 & 23 & 6000.0 & 0 \\
\hline 5 & 8 & 23 & 6000.0 & 0 \\
\hline 54 & 57 & 23 & 4000.0 & 0 \\
\hline 55 & 62 & 23 & 2000.0 & 0 \\
\hline 56 & 66 & 23 & 2000.0 & 0 \\
\hline 72 & 73 & 23 & 2000.0 & 0 \\
\hline 72 & 74 & 23 & 2000.0 & 0 \\
\hline 77 & 78 & 23 & 4000.0 & 0 \\
\hline 77 & 80 & 23 & 2000.0 & 0 \\
\hline 4 & 7 & 23 & 2000.0 & 0 \\
\hline 6 & 12 & 23 & 4000.0 & 0 \\
\hline 8 & 13 & 23 & 4000.0 & 0 \\
\hline 57 & 58 & 23 & 2000.0 & 0 \\
\hline 57 & 59 & 23 & 8000.0 & 0 \\
\hline 62 & 63 & 23 & 2000.0 & 0 \\
\hline 62 & 64 & 23 & 8000.0 & 0 \\
\hline 66 & 67 & 23 & 4000.0 & 0 \\
\hline 74 & 75 & 23 & 2000.0 & 0 \\
\hline 74 & 76 & 23 & 3000.0 & 0 \\
\hline 78 & 79 & 23 & 2000.0 & 0 \\
\hline 78 & 81 & 23 & 100.0 & 1 \\
\hline 7 & 9 & 23 & 2000.0 & 0 \\
\hline 7 & 10 & 23 & 2000.0 & 0 \\
\hline 7 & 11 & 23 & 2000.0 & 0 \\
\hline 12 & 17 & 23 & 4000.0 & 0 \\
\hline 12 & 18 & 23 & 4000.0 & 0 \\
\hline 13 & 19 & 23 & 4000.0 & 0 \\
\hline 13 & 20 & 23 & 4000.0 & 0 \\
\hline 59 & 60 & 23 & 4000.0 & 0 \\
\hline 59 & 61 & 23 & 4000.0 & 0 \\
\hline 64 & 65 & 23 & 4000.0 & 0 \\
\hline 67 & 68 & 23 & 4000.0 & 0 \\
\hline 81 & 82 & 23 & 6000.0 & 0 \\
\hline 28 & 33 & 23 & 4000.0 & 0 \\
\hline 11 & 14 & 23 & 4000.0 & 0 \\
\hline 11 & 15 & 23 & 4000.0 & 0 \\
\hline 11 & 16 & 23 & 2000.0 & 0 \\
\hline 35 & 38 & 23 & 2000.0 & 0 \\
\hline 68 & 69 & 23 & 4000.0 & 0 \\
\hline 68 & 70 & 23 & 6000.0 & 0 \\
\hline 82 & 83 & 23 & 3000.0 & 0 \\
\hline 82 & 86 & 23 & 4000.0 & 0 \\
\hline 24 & 28 & 23 & 2000.0 & 0 \\
\hline 28 & 32 & 23 & 2000.0 & 0 \\
\hline 16 & 21 & 23 & 2000.0 & 0 \\
\hline 16 & 22 & 23 & 2000.0 & 0 \\
\hline 16 & 23 & 23 & 2000.0 & 0 \\
\hline
\end{tabular}


Tabela A.3b - Dados de Ligações do Sistema da Fig. 3 (* - tipos de ligações: 0-trecho sem chave, 1-trecho c/ chave NF, 2-trecho c/ chave NA)

\begin{tabular}{|c|c|c|c|c|}
\hline \multicolumn{2}{|l}{$\begin{array}{l}\text { Nós } \\
\text { terminais }\end{array}$} & Cabo & $\begin{array}{c}\text { Comprim. } \\
(\mathrm{m})\end{array}$ & $\begin{array}{c}\text { Tipo } \\
(*)\end{array}$ \\
\hline 30 & 36 & 23 & 2000.0 & 0 \\
\hline 29 & 35 & 23 & 2000.0 & 0 \\
\hline 35 & 39 & 23 & 3000.0 & 0 \\
\hline 35 & 40 & 23 & 2000.0 & 0 \\
\hline 83 & 84 & 23 & 2000.0 & 0 \\
\hline 83 & 85 & 23 & 2000.0 & 0 \\
\hline 23 & 27 & 23 & 100.0 & 1 \\
\hline 26 & 30 & 23 & 2000.0 & 0 \\
\hline 30 & 37 & 23 & 4000.0 & 0 \\
\hline 25 & 29 & 23 & 4000.0 & 0 \\
\hline 29 & 34 & 23 & 2000.0 & 0 \\
\hline 40 & 46 & 23 & 100.0 & 1 \\
\hline 27 & 31 & 23 & 2000.0 & 0 \\
\hline 37 & 41 & 23 & 1000.0 & 0 \\
\hline 37 & 42 & 23 & 1000.0 & 0 \\
\hline 46 & 48 & 23 & 4000.0 & 0 \\
\hline 31 & 43 & 23 & 2000.0 & 0 \\
\hline 31 & 44 & 23 & 2000.0 & 0 \\
\hline 31 & 45 & 23 & 2000.0 & 0 \\
\hline 48 & 50 & 23 & 3000.0 & 0 \\
\hline 45 & 47 & 23 & 100.0 & 1 \\
\hline 47 & 49 & 23 & 4000.0 & 0 \\
\hline 49 & 51 & 23 & 2000.0 & 0 \\
\hline 49 & 52 & 23 & 4000.0 & 0 \\
\hline 24 & 20 & 23 & 100.0 & 1 \\
\hline 23 & 26 & 23 & 100.0 & 1 \\
\hline 23 & 25 & 23 & 100.0 & 1 \\
\hline 1 & 2 & 23 & 100.0 & 1 \\
\hline 1 & 3 & 23 & 100.0 & 1 \\
\hline 1 & 5 & 23 & 100.0 & 1 \\
\hline 53 & 54 & 23 & 100.0 & 1 \\
\hline 53 & 55 & 23 & 100.0 & 1 \\
\hline 53 & 56 & 23 & 100.0 & 1 \\
\hline 71 & 72 & 23 & 100.0 & 1 \\
\hline 71 & 77 & 23 & 100.0 & 1 \\
\hline 80 & 33 & 23 & 100.0 & 2 \\
\hline 64 & 38 & 23 & 100.0 & 2 \\
\hline 20 & 36 & 23 & 100.0 & 2 \\
\hline 15 & 19 & 23 & 100.0 & 2 \\
\hline 13 & 76 & 23 & 100.0 & 2 \\
\hline 14 & 17 & 23 & 100.0 & 2 \\
\hline 12 & 61 & 23 & 100.0 & 2 \\
\hline 18 & 34 & 23 & 100.0 & 2 \\
\hline 43 & 39 & 23 & 100.0 & 2 \\
\hline 85 & 52 & 23 & 100.0 & 2 \\
\hline 48 & 59 & 23 & 100.0 & 2 \\
\hline 86 & 70 & 23 & 100.0 & 2 \\
\hline & & & & 2 \\
\hline
\end{tabular}

